\title{
Effect of Nitric Oxide on Proline Metabolism in Cucumber Seedlings under Salinity Stress
}

\author{
Huai-Fu Fan ${ }^{1}$ and Chang-Xia Du ${ }^{1}$ \\ Key Laboratory of Southern Vegetable Crop Genetic Improvement, Ministry of Agriculture, College of \\ Horticulture, Nanjing Agricultural University, Nanjing 210095, People's Republic of China; and \\ School of Agriculture and Food Science, Zhejiang Agriculture and Forestry University, Lin'an \\ 311300, People's Republic of China \\ Shi-Rong Guo ${ }^{2}$ \\ Key Laboratory of Southern Vegetable Crop Genetic Improvement, Ministry of Agriculture, College of \\ Horticulture, Nanjing Agricultural University, Nanjing 210095, People's Republic of China
}

\begin{abstract}
Additional Index words. $\mathrm{NaCl}$ stress, Cucumis sativus, exogenous substance, pyrroline-5-carboxylate synthetase, proline dehydrogenase

Abstract. Nitric oxide (NO), an endogenous signaling molecule in plants and animals, mediates responses to abiotic and biotic stresses. This study was conducted in a nutrient solution to investigate 1) the effects of exogenous sodium nitroprusside (SNP), an NO donor, on free proline (Pro) and protein content; and 2) the enzymes involved in Pro metabolism [pyrroline-5-carboxylate synthetase (P5CS) and proline dehydrogenase (PDH)] in cucumber (Cucumis sativus) seedling leaves and roots under $\mathrm{NaCl}$ stress. The results showed that the increases in free Pro and protein were significantly higher in the $50 \mathrm{~mm} \mathrm{NaCl}$ solution but highly significant with the addition of $100 \mu \mathrm{M}$ SNP to the $50 \mathrm{~mm}$ $\mathrm{NaCl}$ solution for the entire treatment period. Moreover, leaves maintained higher levels of free Pro and protein content than roots throughout the experiments. The P5CS activity increased in the saline treatment compared with the control, and this increase was greater in the $50 \mathrm{~mm} \mathrm{NaCl}+100 \mu \mathrm{M}$ SNP solution than in the other treatments. On the other hand, the $\mathrm{PDH}$ activity was inhibited under $\mathrm{NaCl}$ stress but the reduction in activity was greater in the $50 \mathrm{~mm}$ $\mathrm{NaCl}+100 \mu \mathrm{M}$ SNP solution than in the others. These findings suggest that Pro metabolism was significantly altered during the exogenously applied NO under salt stress and that this alteration prompted the accumulation of higher levels of free Pro, which, in turn, maintained the turgor in the cucumber seedlings and helped protect them from salt stress. Moreover, the toxic effects generated by $50 \mathrm{~mm}$ NaCl were partially overcome by the application of NO, which could be used as a potential growth regulator to improve plant salinity tolerance. Therefore, it was concluded that NO could alleviate salinity damage in cucumber seedlings by regulating Pro metabolism. Overall, the adverse effects of salt stress could be lessened by the exogenous application of NO to cucumber seedlings.
\end{abstract}

Salinity is a major environmental factor limiting plant growth, productivity, and distribution. Salinity stress can trigger complex physiological and biochemical responses and affect almost all processes in plants. Under salt stress, cells experience water deficiency, or osmotic stress. Numerous studies have shown that plants under salt stress can actively accumulate small molecule metabolites and inorganic ions, which contribute to growth and development in plants. However, soluble metabolites are more important than inorganic ions for plants when they suffer environmental stresses. Proline, glycine betaine, and soluble sugars are examples of low-molecular-weight solutes that accumulate in plants. Pro is one of the most commonly accumulated osmolytes in plants under salinity conditions (Delauney and Verma, 1993; Misra and Saxena, 2009), and it is often considered to be involved in stress resistance mechanisms. In addition to its role as an osmolyte in osmotic adjustment (Ashraf and Foolad, 2007), it has been suggested that Pro stabilizes subcellular structures,

Received for publication 26 Jan. 2012. Accepted for publication 29 Mar. 2012. This research was supported by the National Basic Research Program of China (973 Program; No. 2009CB119000), the National Nature Science Foundation of China (No. 30871736, 30900995, and 31101539), and the Earmarked Fund for Modern Agro-industry Technology Research System (No. Nycytx-35-gw18).

${ }^{1}$ Equal contributions to this article.

${ }^{2}$ Corresponding author. E-mail: srguo@njau.edu.cn. scavenges free radicals (Chris et al., 2006), and buffers the cellular redox potential under stress conditions (Ashraf and Foolad, 2007; Gomes et al., 2010). Pro also functions as an energy sink in the regulation of redox potentials and alleviates salt stress-induced enhancement in oxygenase and carboxylase activities of ribulose-1,5-bisphosphate carboxylase-oxygenase (Sivakumar et al., 2000). Although the role of Pro accumulation in stress resistance is highly controversial, it is hypothesized that high levels of Pro can be beneficial to stressed plants (Yazici et al., 2007). An increasing number of studies have shown that the accumulation of Pro is positively correlated with stress tolerance in plants (Hanson et al., 1977; Hong et al., 2000). Many researchers propose that Pro accumulation in plants is a protective countermeasure under salt stress and potentially a salt stress signal (Khedr et al., 2003; Szabados and Savouré, 2009). In addition, the intermediates of Pro metabolism induce gene expression and reduce oxidation injuries from osmotic stress (Hong et al., 2000). Although Pro-inducible genes have been identified (Satoh et al., 2002), the mechanisms of Pro action are not fully understood. Pro is synthesized in plants through two pathways: the glutamate (Glu) approach and the ornithine approach by original amino acids. Pro biosynthesis from Glu appears to be the predominant pathway, especially under stress conditions (Delauney and Verma, 1993). The enzyme pyrroline5 -carboxylate synthetase plays a key role in controlling the level 
of Pro in plants and catalyzes the first two steps of Pro biosynthesis from Glu (Hu et al., 1992). P5CS has been isolated from several plants (Fujita et al., 1998; Hu et al., 1992; Yoshiba et al., 1995), and a correlation between the induction of P5CS and the accumulation of Pro has been shown in Arabidopsis thaliana and rice [Oryza sativa (Yoshiba et al., 1995, 1997)]. The Procatabolizing enzymes Pro oxidase and proline dehydrogenase also influence the level of Pro accumulation. Greater accumulation of Pro could be caused by the inhibition of these enzymes (Kandpal et al., 1981).

NO is a small, highly diffusible, gaseous-free radical and a ubiquitous bioactive molecule that plays a key role as an intraand intercellular messenger inducing various processes through either redox or additive chemistry in plants (Lamattina et al., 2003). These processes include senescence (Leshem et al., 1998), stress responses (Ruan et al., 2004), programmed cell death (Beligni et al., 2002), and disease resistance (Chandok et al., 2003). Along with other plant growth regulators, however, NO is a reactive nitrogen species, and studies have shown its effects on different cells are either protective or toxic, depending on its concentration and the position of action (Lamattina et al., 2003). In recent years, increasing evidence has demonstrated that NO serves as a signal in developmental, hormonal, and stress responses in plants at lower concentration (Beligni et al., 2002; He et al., 2004; Xu et al., 2010). In relation to abiotic stresses, application of the NO donor sodium nitroprusside has been shown to reduce the harmful effects of salinity on plants (Fan et al., 2010). Furthermore, NO is able to scavenge reactive oxygen species (ROS) as an antioxidant agent and alter antioxidative gene expression as a signaling molecule, thus protecting plant cells from oxidative damage (Arasimowicz and Floryszak-Wieczorek, 2007). In our previous studies, NO was shown to alleviate salt stress in cucumber seedlings by regulating free polyamines and scavenging free radicals (Fan et al., 2010). Pro levels are correlated with NO because L-arginine is a common precursor in their biosynthesis (Gao et al., 2009). The effects of exogenously applied NO on Pro accumulation and the metabolism of plants grown under salt stress are still unclear. Therefore, cucumber, an important horticultural crop, was selected as the test plant for this study because it is highly sensitive to salinity, especially during germination and the seedling stage. In this study, we investigated the effects of NO on Pro content and related enzymes' activities in cucumber seedlings under $\mathrm{NaCl}$ stress. The main goals of this research were to study the effects of exogenous NO application on Pro metabolism in cucumber seedlings grown under salt stress and to elucidate the physiological mechanism of the increased tolerance of cucumber plants to salt stress resulting from the exogenous application of NO.

\section{Materials and Methods}

The experiments were carried out in a greenhouse at Nanjing Agricultural University, Nanjing, China. Cucumber (cv. Jinchun 2) seeds were placed in sterile petri plates on filter paper moistened with distilled water. They were allowed to germinate in the dark in a thermostatically controlled chamber at $29 \pm 1{ }^{\circ} \mathrm{C}$ for $\approx 30 \mathrm{~h}$. The germinated seeds were sown in washed quartz sands. The average temperatures under natural light were 25 to $30{ }^{\circ} \mathrm{C}$ during the day and 16 to $20{ }^{\circ} \mathrm{C}$ at night. Relative humidity fluctuated between $60 \%$ and $70 \%$. At the second fully expanded leaf stage (at $21 \mathrm{~d}$ after sowing), the cucumber leaves were numbered from the apical to the basal nodes. Seedlings were removed from the plastic plates, and the roots were rinsed with distilled water. Uniformly sized healthy seedlings were selected and transferred into troughs $(51 \times 33 \times 20 \mathrm{~cm})$ filled with $20 \mathrm{~L}$ fullstrength Hoagland's nutrient solution, which was aerated for 40 min each hour. After pre-culturing for $3 \mathrm{~d}$, the seedlings were treated with one of the following methods: 1) control [CK (full-strength Hoagland's nutrient solution)]; 2) $\mathrm{NaCl}$ treatment $[\mathrm{NaCl}$ (full-strength Hoagland's nutrient solution containing $50 \mathrm{~mm} \mathrm{NaCl}$ )]; 3) SNP treatment $[\mathrm{NaCl}+\mathrm{SNP}$ (full-strength Hoagland's nutrient solution containing $50 \mathrm{~mm}$ $\mathrm{NaCl}$ with $100 \mu \mathrm{M}$ SNP)]; or 4) $\mathrm{NaNO}_{2}$ treatment $[\mathrm{NaCl}+$ $\mathrm{NaNO}_{2}$ (full-strength Hoagland's nutrient solution containing $50 \mathrm{~mm} \mathrm{NaCl}$ with $\left.1 \mu \mathrm{M} \mathrm{NaNO} \mathrm{N}_{2}\right)$ ]. SNP was used as the NO donor. The $\mathrm{NaNO}_{2}$ treatment served as a second control because the decomposition of $100 \mu \mathrm{M}$ SNP generates a maximum of $1 \mu \mathrm{M} \mathrm{NO}_{2}^{-}$as a byproduct. Troughs were arranged in a completely randomized block design with three replicates for each treatment (for a total of 12 troughs). All the nutrient solutions were renewed every $2 \mathrm{~d}$ to maintain identical concentrations.

Leaf and root samples from healthy cucumber seedling were harvested in triplicate at $0,2,4,6$, and $8 \mathrm{~d}$ after initiation of the treatment and analyzed immediately.

Free Pro content was determined according to Bates et al. (1973). Approximately $0.5 \mathrm{~g}$ of leaf or root samples from each group was homogenized in 3\% (w/v) sulphosalycylic acid, and the homogenate was filtered through filter paper. After adding acid ninhydrin and glacial acetic acid, the resulting mixture was heated at $100{ }^{\circ} \mathrm{C}$ for $1 \mathrm{~h}$ in a water bath. The reaction was then stopped with an ice bath. The mixture was extracted with toluene, and the absorbance of the fraction with the toluene aspired from the liquid phase was read at $520 \mathrm{~nm}$. Pro concentration was measured with a calibration curve and expressed as micrograms of Pro per gram fresh weight.

P5CS activity was assayed according to the method of Vogel and Kopac (1960) modified as follows. Approximately $0.7 \mathrm{~mL}$ of reaction medium containing $50 \mathrm{~mm}$ Tris $(\mathrm{pH} 7.5), 2 \mathrm{~mm}$ $\mathrm{MgCl}_{2}, 10 \mathrm{~mm}$ ATP, $1 \mathrm{~mm} \mathrm{NADH}, 50 \mathrm{~mm}$ glutamic acid, and $0.1 \mathrm{~mL}$ of enzyme extract was incubated at $37^{\circ} \mathrm{C}$ for $30 \mathrm{~min}$. The reaction was then stopped with $0.3 \mathrm{~mL}$ of $10 \%(\mathrm{w} / \mathrm{v})$ trichloroacetic acid. The color reaction was developed by incubating the solution with $0.1 \mathrm{~mL}$ of $0.5 \%(\mathrm{w} / \mathrm{v})$ aminobenzaldehyde for $1 \mathrm{~h}$. After centrifugation at $12,000 g_{\mathrm{n}}$ for $10 \mathrm{~min}$, the clear supernatant fraction was removed and the absorbance at $440 \mathrm{~nm}$ was measured. A 0.1 absorbance change in optical density at $440 \mathrm{~nm} \cdot \mathrm{h}^{-1}$ was defined as one unit of enzyme activity.

For the extraction and assay of $\mathrm{PDH}$, fresh leaves or roots $(0.5 \mathrm{~g})$ were homogenized in $50 \mathrm{~mm}$ Tris- $\mathrm{HCl}$ buffer containing $7 \mathrm{mM} \mathrm{MgCl}_{2}, 0.6 \mathrm{M} \mathrm{KCl}, 3 \mathrm{~mm}$ EDTA, $1 \mathrm{~mm}$ dithiothreitol, and $5 \%(\mathrm{w} / \mathrm{v})$ polyvinylpolypyrrolidone, crosslinked, and adjusted to a $\mathrm{pH}$ of 7.4. The homogenate was filtered and centrifuged at $39,000 g_{n}$ for $20 \mathrm{~min}$ at $4{ }^{\circ} \mathrm{C}$ (Rosales et al., 2007). The supernatant was used to determine $\mathrm{PDH}$ activity. $\mathrm{PDH}$ activity was assayed by reduction of $\mathrm{NAD}^{+}\left(\right.$or $\mathrm{NADP}^{+}$) at $340 \mathrm{~nm}$. The reaction mixture had a $\mathrm{pH}$ of 10.3 and contained $0.15 \mathrm{mM} \mathrm{Na}_{2} \mathrm{CO}_{3}$ $\mathrm{HCl}$ buffer, $15 \mathrm{~mm}$ L-proline, and $1.5 \mathrm{mM} \mathrm{NAD}^{+}$or $\mathrm{NADP}^{+}$(Miller and Stewart, 1976).

Protein was measured following the procedure of Bradford (1976) with bovine serum albumin as the standard.

All experiments were performed in triplicate. All data were statistically analyzed with SAS (Version 8.2; SAS Institute, Cary, NC) using Duncan's multiple range test at the 5\% level of significance. 


\section{Results}

Pro content. As shown in Figure 1A, the Pro content in the cucumber seedling leaves increased significantly under $\mathrm{NaCl}$ stress compared with the CK; Pro content was $146 \%, 196 \%$, $299 \%$, and $356 \%$ of the CK plant content at 2, 4, 6, and $8 \mathrm{~d}$ of treatment, respectively. Exogenous supply of SNP further increased the Pro content. The Pro content in the leaves of the cucumber seedlings grown in the SNP treatment reached the highest level at $8 \mathrm{~d}$ of treatment and was $12 \%$ higher than that grown under $\mathrm{NaCl}$ stress alone.

Similarly, $\mathrm{NaCl}$ stress significantly increased the Pro content of the roots (Fig. 1B). However, the leaves always maintained a higher level of Pro than the roots. A highly significant increase was observed in SNP $+\mathrm{NaCl}$ as compared with other treatments. Although the difference of Pro content between the salt treatment and the SNP treatment decreased after $8 \mathrm{~d}$ of treatment, the difference remained obvious.

Compared with $\mathrm{NaCl}$ stress, the application of $\mathrm{NaNO}_{2}$ to the salinized solution had no obvious effect on the Pro content in the leaves or roots.
Pyrroline-5-Carboxylate Synthetase activity. Salt stress caused an increase of the P5CS activity in the cucumber seedling leaves as represented in Figure 2A. SNP treatment brought out higher P5CS activity over the $\mathrm{NaCl}$ stress alone. The P5CS activity in the leaves treated with $\mathrm{SNP}+\mathrm{NaCl}$ was significantly higher than that under $\mathrm{NaCl}$ stress alone at 6 and $8 \mathrm{~d}$ of treatment. The application of $\mathrm{NaNO}_{2}$ to the salinized solution produced an P5CS activity similar to that produced under $\mathrm{NaCl}$ treatment alone throughout the treatment period (Fig. 2A).

The P5CS activity of the cucumber roots increased under $\mathrm{NaCl}$ stress, which was significantly higher than that under CK conditions at 4 and $8 \mathrm{~d}$ of treatment. Application of SNP increased the P5CS activity in the roots under $\mathrm{NaCl}$ stress, and with SNP in solution, the activity in the roots under $\mathrm{NaCl}$ stress was significantly higher than that under $\mathrm{NaCl}$ stress alone at 4,6 , and $8 \mathrm{~d}$. Combining $50 \mathrm{~mm} \mathrm{NaCl}$ with $1 \mu \mathrm{M} \mathrm{NaNO}_{2}$ in solution had no obvious effects on the P5CS activity in the roots (Fig. 2B).

Proline dehydrogenase activity. Figure 3A depicted the PDH activity in leaves grown under various treatments. The PDH activity in the leaves decreased during exposure to salinity.

$\square \mathrm{CK} \square \mathrm{NaCl} \square \mathrm{NaCl}+\mathrm{SNP} \quad \mathbf{\mathrm { NaCl }}+\mathrm{NaNO} 2$

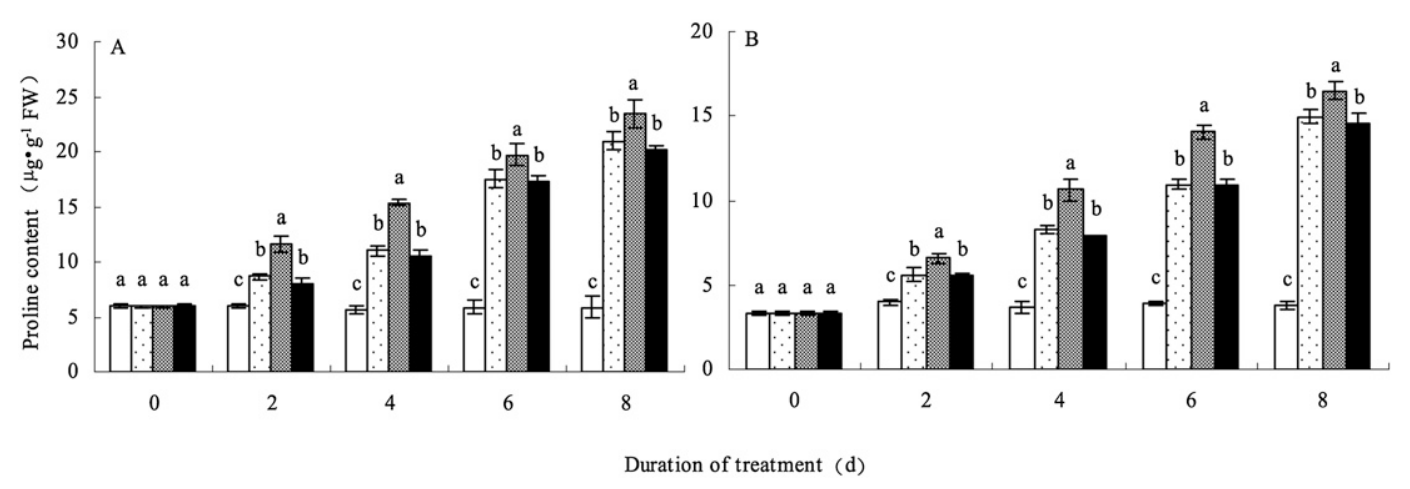

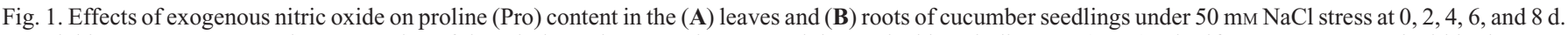
Each histogram represents the mean value of three independent experiments, and the vertical bars indicate $\mathrm{SE}(\mathrm{n}=3)$. Significances were tested within the same day. Different letters indicate significant differences between treatments through Duncan's multiple range test at $P<0.05$; CK $=$ control; SNP $=$ sodium nitroprusside.

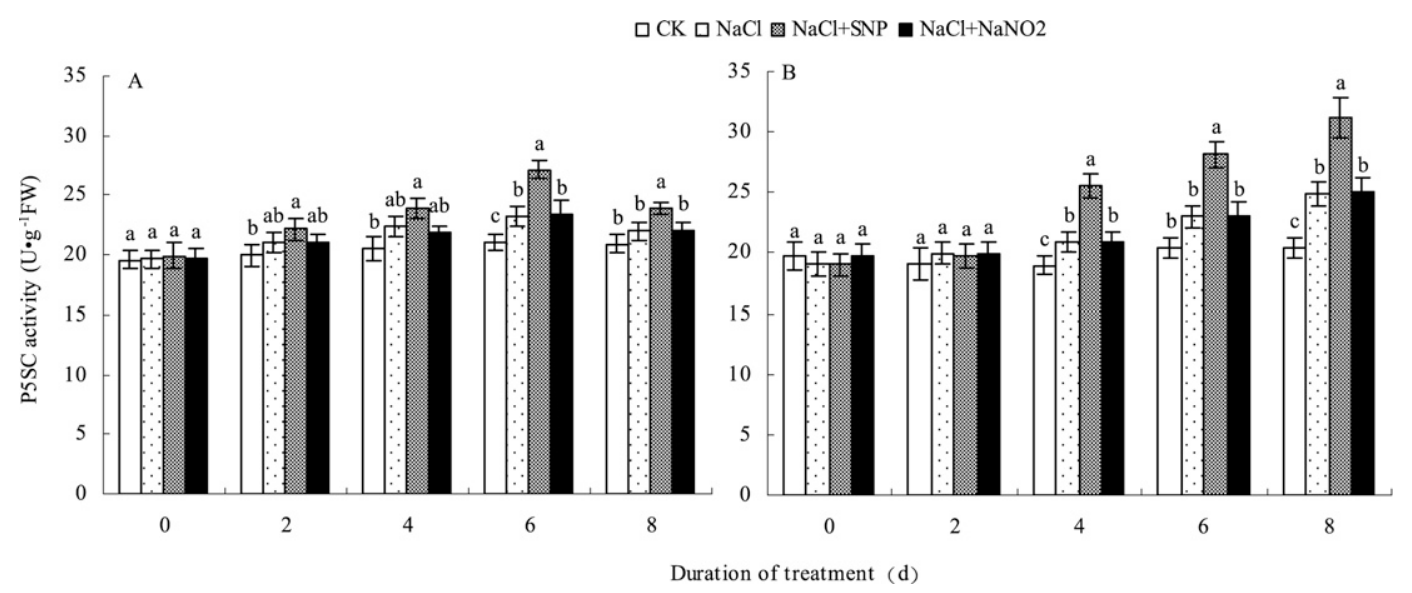

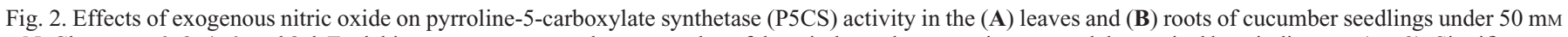
$\mathrm{NaCl}$ stress at $0,2,4,6$, and $8 \mathrm{~d}$. Each histogram represents the mean value of three independent experiments, and the vertical bars indicate sE ( $=3$ ). Significances were tested within the same day. Different letters indicate significant differences between treatments through Duncan's multiple range test at $P<0.05$; CK $=$ control; SNP = sodium nitroprusside. 
The difference between the $\mathrm{NaCl}$ treatment and the $\mathrm{CK}$ at 4,6 , and $8 \mathrm{~d}$ was significant. The addition of SNP to the salinized nutrient solution reduced the $\mathrm{PDH}$ activity throughout the treatment period. Applying $\mathrm{NaNO}_{2}$ to the salinized solution had no obvious effect on the PDH activity in the leaves.

The PDH activity in the roots of the cucumber seedlings showed a similar trend to that in the leaves. As compared with plants grown in $\mathrm{CK}$ solution, the PDH activity in the roots under salt stress decreased throughout the treatment period and reached a significant level at 4, 6, and $8 \mathrm{~d}$. Application of SNP to the salinized nutrient solution decreased the PDH activity throughout the treatment period, and the PDH activity was markedly lower than that under the salt treatment alone at $2 \mathrm{~d}$. The PDH activity was higher in the roots than in the leaves throughout the treatment period. Combining $50 \mathrm{~mm} \mathrm{NaCl}$ with $1 \mu \mathrm{M} \mathrm{NaNO}_{2}$ in solution had no obvious effects on the $\mathrm{PDH}$ activity in the roots (Fig. 3B).

Protein Content. The protein content in the leaves markedly increased under salt stress and continued to increase throughout the treatment period (Fig. 4A). The addition of SNP to the salinized nutrient solution increased the protein content, which was $145 \%, 145 \%, 111 \%$, and $116 \%$ of that under $\mathrm{NaCl}$ stress alone at 2, 4, 6, and $8 \mathrm{~d}$, respectively. Applying $\mathrm{NaNO}_{2}$ to the salinized solution had no obvious effect on the protein content in the leaves.
Figure 4B showed the protein content in the roots of the cucumber seedlings in $\mathrm{CK}$ and in the presence of various treatments. The protein content in the roots increased under salt stress throughout the treatment period. The addition of SNP to the salinized nutrient solution clearly elevated the protein content. The protein content was higher in the leaves than in the roots throughout the treatment period. Combining $50 \mathrm{~mm} \mathrm{NaCl}$ with $1 \mu \mathrm{M} \mathrm{NaNO}{ }_{2}$ in solution had no obvious effects on the protein content in the roots.

\section{Discussion and Conclusions}

The mechanisms of salt tolerance are not yet clear, but they can be partially explained by stress-adaptation effectors that mediate ion homeostasis, osmolyte biosynthesis, ROS scavenging, water transport, and long-distance response coordination (Hasegawa et al., 2000). The typical first response of all plants to salt stress is osmotic adjustment. The accumulation of compatible solutes in the cytoplasm is considered to be a mechanism that contributes to salt tolerance (Jaleel et al., 2007). To counter salt stress, plants decrease the osmotic potential of their cells by synthesizing and accumulating compatible osmolytes such as Pro, which participate in osmotic adjustment (Ghoulam et al., 2002). The accumulation of Pro under stress in many plant species has been correlated with stress tolerance (Ashraf and Foolad,

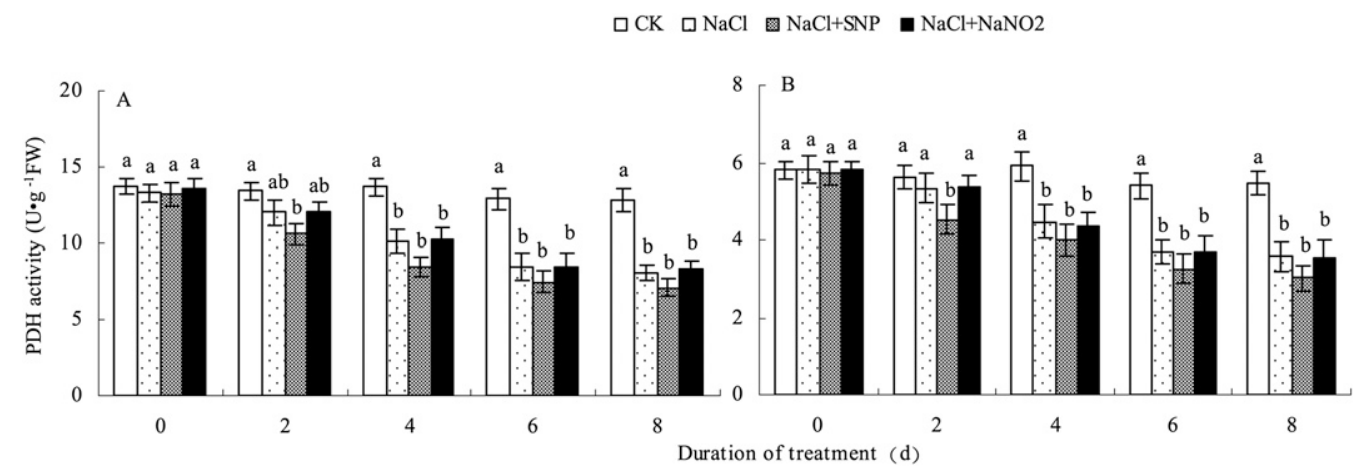

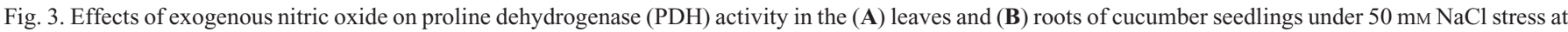
$0,2,4,6$, and $8 \mathrm{~d}$. Each histogram represents the mean value of three independent experiments, and the vertical bars indicate SE $(\mathrm{n}=3)$. Significances were tested within the same day. Different letters indicate significant differences between treatments through Duncan's multiple range test at $P<0.05$; CK $=$ control; $\mathrm{SNP}=$ sodium nitroprusside.

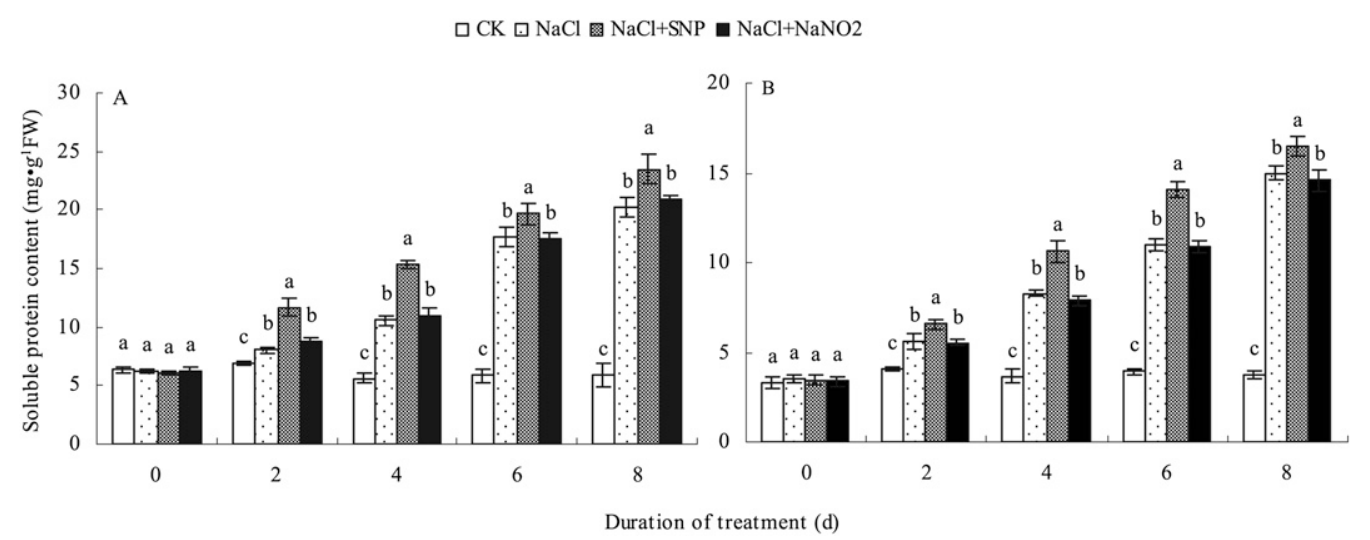

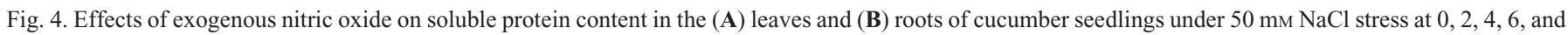
$8 \mathrm{~d}$. Each histogram represents the mean value of three independent experiments, and the vertical bars indicate sE ( $\mathrm{n}=3$ ). Significances were tested within the same day. Different letters indicate significant differences between treatments through Duncan's multiple range test at $P<0.05$; $\mathrm{CK}=\mathrm{control}$; SNP $=$ sodium nitroprusside. 
2007), and the concentration of Pro has been shown to be higher in stress-tolerant than in stress-sensitive plants (Demiral and Türkan, 2006). Giannakoula et al. (2008) verified the role of Pro in the protection of plants against abiotic stress; tolerant plant lines, which had higher Pro levels, suffered less damage than sensitive plant lines, which had lower Pro levels. It has also been shown that NO improves the salt tolerance of many plant species (Zhang et al., 2006). In our previous studies, we showed that NO-induced enhancement in plants grown under salinity stress might have been caused by NO-induced changes in physiological processes. NO-induced increase in growth could be related to the enhanced activity of antioxidants, which protect plants from oxidative damage (Fan et al., 2010). Ruan et al. (2004) reported that exogenous NO enhanced salt tolerance by increasing the Pro content in leaves of wheat (Triticum aestivum). Our studies showed that NO also increased the Pro content in cucumber leaves and roots under salt stress. We conclude that NO enhanced the salt tolerance of cucumber seedlings by increasing the Pro content.

Pro accumulation in plant tissues has been attributed to 1) an increase in Pro biosynthesis; 2) a decrease in Pro degradation; 3) a decrease in protein synthesis or Pro use; and 4) the hydrolysis of proteins (Ashraf and Foolad, 2007). Hare et al. (1999) described in detail the molecular regulation mechanisms of Pro synthesis and degradation. Under salt stress, Pro is synthesized mainly through the glutamic acid pathway. Glu is the major amino acid involved in Pro synthesis, and transgenic tobacco (Nicotiana tabacum) plants with a reduced expression of cytosolic glutamine synthetase accumulated less Pro than non-transformed plants under salt stress (Kavikishore et al., 1995). P5CS is a rate-limiting enzyme for the biosynthetic pathway in higher plants and is feedback-inhibited by Pro (Zhang et al., 1995). In this pathway, Pro is synthesized from Glu through P5C by two successive reductions, which are catalyzed by P5CS and P5C reductase (Hare et al., 1999). High levels of Pro synthesis in stressed plants under field conditions could favor a better recovery. Also, it has been reported that higher Pro accumulation in P5CS-transformed tobacco plants reduces free radical levels, as measured by malondialdehyde content, in response to osmotic stress (Parvanova et al., 2004). Transgenic tobacco plants overexpressing P5CS have shown an increased concentration of Pro and resistance to both drought and salinity stresses (Kishor et al., 1995). Salt stress, dehydration stress, or abscisic acid can induce the expression of P5CS (Silva-Ortega et al., 2008; Sripinyowanich et al., 2010). Vendruscolo et al. (2007) reported the effects of water deficit on wheat plants transformed with the Vigna aconitifolia P5CS cDNA and found that the tolerance to water deficit in transgenic plants was mainly the result of mechanisms that protect plants against oxidative stress, not to osmotic adjustment. Uchida et al. (2002) proved that SNP induced the mRNA expression of P5CS by a Northern blot analysis, and this provided the basis for our research on the adjustment of Pro metabolism under salt stress. In this study, we found that salt stress improved the P5CS activity of cucumber, and NO further increased P5CS activity in leaves and roots, in accordance with Ruan et al. (2004). Hong et al. (2000) demonstrated that the feedback regulation of $\mathrm{P} 5 \mathrm{CS}$ played a role in controlling the level of Pro in plants under both normal and stress conditions. This contradicts the continued synthesis of Pro by plants under stress. P5CS is subject to allosteric regulation by Pro (Hu et al., 1992; Zhang et al., 1995). The inhibition of feedback regulation of $\mathrm{P} 5 \mathrm{CS}$ is lost during stress, and this increases
Pro accumulation. We observed an increase in P5CS activity and Pro content only under salt stress alone or under salt stress with SNP in solution.

Conversely, Pro content also depends on its degradation (Yoshiba et al., 1997). PDH, the key enzyme that catalyzes Pro degradation in mitochondria, acts from transcriptional expression to protein synthesis and finally catalyzes Pro into P5C, which is then converted into glutamic acid by P5C dehydrogenase (P5CDH). These steps constitute the principal processes of oxidative Pro degradation (Hare et al., 1999). In A. thaliana, AtPDH was the only enzyme acting as a functional PDH (Ashraf and Foolad, 2007). Our studies showed that exogenous NO reduced the PDH activity in cucumber leaves and roots, resulting in a reduction of Pro degradation in cucumber seedlings under salt stress. In response to water stress, Pro accumulation is dependent on the transcriptional activation and translation of NAD $(\mathrm{P}) \mathrm{H}$ dependent P5CS to produce P5C (Yoshiba et al., 1995). Furthermore, Pro degradation is controlled by $\mathrm{PDH}$ and P5CDH. Parre et al. (2007) identified the signaling components involved in the regulation of Pro metabolism under water stress in A. thaliana and indicated that PLC (phosphoinositide-specific phospholipase C)-based signaling was a committed step in Pro biosynthesis under salinity stress but not under mannitol stress.

Our results showed that the Pro content in leaves was higher than that in roots based on a combination of the changing trends of P5CS and PDH activity. This could be attributed to serious metabolic problems such as deficits of substrates and intermediates involved in Pro synthesis, caused by the presence of salt on the outside of roots in the nutrient solution, a condition to which leaves are subjected indirectly. Thus, we observed that the P5CS activity in roots was higher than that in leaves and that the PDH activity in roots was lower than that in leaves. In contrast, Pro accumulation was higher in leaves than in roots.

Exogenous NO increased the protein content in leaves and roots and thereby increased salt tolerance. With its hydrophobic end proteins and hydrophilic end-binding hydrones, Pro has a strong hydrating ability. This forces the proteins to bind more water molecules, preventing dehydration and denaturation (Hoekstra et al., 2001). A small increase in Pro could be caused by the hydrolysis of Pro-rich proteins (Song et al., 2005). We observed protein and Pro accumulation, indicating that the accumulation of Pro may come from the hydrolysis of Pro-rich proteins under salt stress. Moreover, protein accumulation was not caused by the use of Pro for protein synthesis. Buhl and Stewart (1983) also found that salt treatment dramatically increased the synthesis of Pro from Glu and that its use in oxidation and protein synthesis decreased by $50 \%$ and $60 \%$, respectively, in barley (Hordeum vulgare) under salinity stress.

Based on the results from this study, it can be concluded that exogenous SNP increased salt tolerance in cucumber seedlings by adjusting the biosynthesis of Pro; as the P5CS activity increased and the PDH activity decreased, the accumulation of Pro accelerated. SNP could be used as a potential growth regulator to improve plant growth under salinity stress. Furthermore, the application of $\mathrm{NO}_{2}^{-}$(a byproduct from the decomposition of SNP) did not produce effects similar to those of SNP.

\section{Literature Cited}

Arasimowicz, M. and J. Floryszak-Wieczorek. 2007. Nitric oxide as a bioactive signaling molecule in plant stress responses. Plant Sci. 172:876-887. 
Ashraf, M. and M.R. Foolad. 2007. Roles of glycine betaine and proline in improving plant abiotic stress resistance. Environ. Expt. Bot. 59:206-216.

Bates, L.S., R.P. Waldren, and I.D. Teare. 1973. Rapid determination of free proline for water-stress studies. Plant Soil 39:205-207.

Beligni, M.V., A. Fath, P.C. Bethke, L. Lamattina, and R.L. Jones. 2002. Nitric oxide acts as an antioxidant and delays programmed cell death in barley aleurone layers. Plant Physiol. 129:1642-1650.

Bradford, M.M. 1976. A Rapid and sensitive method for the quantitation of microgram quantities of protein utilizing the principle of protein-dye binding. Anal. Biochem. 72:248-254.

Buhl, M.B. and G.R. Stewart. 1983. Effect of $\mathrm{NaCl}$ on proline synthesis and utilization in excised barley leaves. Plant Physiol. 72:664-667.

Chandok, M.R., A.J. Ytterberg, K.J. van Wijk, and D.F. Klessig. 2003. The pathogen-inducible nitric oxide synthase (iNOS) in plants is a variant of the P protein of the glycine decarboxylase complex. Cell 113:469-482.

Chris, A., M. Zeeshan, G. Abraham, and S.M. Prasad. 2006. Proline accumulation in Cylindrospermum sp. Environ. Expt. Bot. 57:154-159.

Delauney, A.J. and D.P.S. Verma. 1993. Proline biosynthesis and osmoregulation in plants. Plant J. 4:215-223.

Demiral, T. and I. Türkan. 2006. Exogenous glycine betaine affects growth and proline accumulation and retards senescence in two rice cultivars under $\mathrm{NaCl}$ stress. Environ. Expt. Bot. 56:72-79.

Fan, H.F., C.X. Du, and S.R. Guo. 2010. Nitric oxide enhances salt tolerance in cucumber seedlings by regulating free polyamine content. Environ. Expt. Bot. doi: 10.1016 /j.envexpbot.2010.09.007. Fujita, T., A. Maggio, M. Garcia-Rios, R.A. Bressan, and L.N. Csonka. 1998. Comparative analysis of the regulation of expression and structures of two evolutionarily divergent genes for $\Delta^{1}$-pyrroline-5carboxylate synthetase from tomato. Plant Physiol. 118:661-674.

Gao, H.J., H.Q. Yang, and J.X. Wang. 2009. Arginine metabolism in roots and leaves of apple (Malus domestica Borkh.): The tissuespecific formation of both nitric oxide and polyamines. Sci. Hort. 119:147-152.

Ghoulam, C., A. Foursy, and K. Fares. 2002. Effects of salt stress on growth, inorganic ions and proline accumulation in relation to osmotic adjustment in five beet cultivars. Environ. Expt. Bot. 47:39-50.

Giannakoula, A., M. Moustakas, P. Mylona, I. Papadakis, and T. Yupsanis. 2008. Aluminum tolerance in maize is correlated with increased levels of mineral nutrients, carbohydrates and proline, and decreased levels of lipid peroxidation and $\mathrm{Al}$ accumulation. J. Plant Physiol. 165:385-396.

Gomes, F.P., M.A. Oliva, M.S. Mielke, A.A.F. Almeida, and L.A. Aquino. 2010. Osmotic adjustment, proline accumulation and cell membrane stability in leaves of Cocos nucifera submitted to drought stress. Sci. Hort. 126:379-384.

Hanson, A.D., C.E. Nelsen, and E.H. Everson. 1977. Evaluation of free proline accumulation as an index of drought resistance using two contrasting barley cultivars. Crop Sci. 17:720-726.

Hare, P.D., W.A. Cress, and J. van Staden. 1999. Proline synthesis and degradation: A model system for elucidating stress-related signal transduction. J. Expt. Bot. 50:413-434.

Hasegawa, P.M., R.A. Bressan, J.K. Zhu, and H.J. Bohnert. 2000. Plant cellular and molecular responses to high salinity. Annu. Rev. Plant Physiol. Plant Mol. Biol. 51:463-499.

He, Y., R.H. Tang, Y. Hao, R.D. Stevens, C.W. Cook, S.M. Ahn, L. Jing, Z. Yang, L. Chen, F. Guo, F. Fiorani, R.B. Jackson, N.M. Crawford, and Z.M. Pei. 2004. Nitric oxide represses the Arabidopsis floral transition. Science 305:1968-1971.

Hoekstra, F.A., E.A. Golovina, and J. Buitink. 2001. Mechanisms of plant desiccation tolerance. Trends Plant Sci. 6:431-438.

Hong, Z., K. Lakkineni, Z. Zhang, and D.P.S. Verma. 2000. Removal of feedback inhibition of $\Delta^{1}$-pyrroline-5-carboxylate synthetase results in increased proline accumulation and protection of plants from osmotic stress. Plant Physiol. 122:1129-1136.

Hu, C.A.A., A.J. Delauney, and D.P.S. Verma. 1992. A bifunctional enzyme ( $\Delta^{1}$-pyrroline-5-carboxylate synthetase) catalyzes the first two steps in proline biosynthesis in plants. Proc. Natl. Acad. Sci. USA 89:9354-9358.

Jaleel, C.A., P. Manivannan, A. Kishorekumar, B. Sankar, and R. Panneerselvam. 2007. Calcium chloride effects on salinity induced oxidative stress, proline metabolism and indole alkaloid accumulation in Catharanthus roseus. C. R. Biol. 330:674-683.

Kandpal, R.P., C.S. Vaidyanathan, M. Udaya Kumar, K.S. Krishna Sastry, and N. Appaji Rao. 1981. Alterations in the activities of the enzyme of proline metabolism in Ragi (Eleusine coracane) leaves during water stress. J. Biosci. 3:361-369.

Kavikishore, P.B., Z. Hong, G.U. Miao, C.A.H. Hu, and D.P.S. Verma. 1995. Over expression of pyrroline-5-carboxylase synthetase increases proline production and confers osmotolerance in transgenic plants. Plant Physiol. 108:1387-1394.

Khedr, A.H.A., M.A. Abbas, A.A.A. Wahid, W.P. Quick, and G.M. Abogadallah. 2003. Proline induces the expression of salt-stressresponsive proteins and may improve the adaptation of Pancratium maritimum L. to salt stress. J. Expt. Bot. 54:2553-2562.

Kishor, P.B.K., Z. Hong, G.H. Miao, C.A.A. Hu, and D.P.S. Verma. 1995. Overexpression of $\Delta^{1}$-pyrroline-5-carboxylate synthetase increases proline production and confers osmotolerance in transgenic plants. Plant Physiol. 108:1387-1394.

Lamattina, L., C. Garica-Mata, M. Graziano, and G. Pagnussat. 2003. Nitric oxide: The versatility of an extensive signal molecule. Annu. Rev. Plant Biol. 54:109-136.

Leshem, Y.Y., R.B.H. Wills, and V.V.V. Ku. 1998. Evidence for the function of the free radical gas - nitric oxide (NO) — as an endogenous maturation and senescence regulating factor in higher plant. Plant Physiol. Biochem. 36:825-833.

Miller, P.M. and C.R. Stewart. 1976. Pyrroline-5-carboxylic acid reductase from soybean leaves. Phytochemistry 15:1855-1857.

Misra, N. and P. Saxena. 2009. Effect of salicylic acid on proline metabolism in lentil grown under salinity stress. Plant Sci. 177: 181-189.

Parre, E., M.A. Ghars, A.S. Leprince, L. Thiery, D. Lefebvre, M. Bordenave, L. Richard, C. Mazars, C. Abdelly, and A. Savouré. 2007. Calcium signaling via phospholipase $\mathrm{C}$ is essential for proline accumulation upon ionic but not nonionic hyperosmotic stresses in Arabidopsis. Plant Physiol. 144:503-512.

Parvanova, D., S. Ivanov, T. Konstantinova, E. Karanov, A. Atanassov, T. Tsvetkov, V. Alexieva, and D. Djilianov. 2004. Transgenic tobacco plants accumulating osmolytes show reduced oxidative damage under freezing stress. Plant Physiol. Biochem. 42:57-63.

Rosales, M.A., J.J. Rios, R. Castellano, A.I. Lopez-Carrion, and I.M. Ruiz. 2007. Proline metabolism in cherry tomato exocarp in relation to temperature and solar radiation. J. Hort. Sci. Biotechnol. 82:739744.

Ruan, H.H., W.B. Shen, and L.L. Xu. 2004. Nitric oxide involved in the abscisic acid induced proline accumulation in wheat seedling leaves under salt stress. Acta Bot. Sin. 46:1307-1315.

Satoh, R., K. Nakashima, M. Seki, K. Shinozaki, and K. YamaguchiShinozaki. 2002. ACTCAT, a novel cis-acting element for prolineand hypoosmolarity-responsive expression of the ProDH gene encoding proline dehydrogenase in Arabidopsis. Plant Physiol. 130: 709-719.

Silva-Ortega, C.O., A.E. Ochoa-Alfaro, J.A. Reyes-Agüero, G.A. Aguado-Santacruz, and J.F. Jiménez-Bremont. 2008. Salt stress increases the expression of $p 5 c s$ gene and induces proline accumulation in cactus pear. Plant Physiol. Biochem. 46:82-92.

Sivakumar, P., P. Sharmila, and P. Parthasaradhi. 2000. Proline alleviates salt stress-induced enhancement in ribulose-1,5-bisphosphate oxygenase activity. Biochem. Biophys. Res. Commun. 279:512-515.

Song, S.Q., Y.B. Lei, and X.R. Tian. 2005. Proline metabolism and cross-tolerance to salinity and heat stress in germinating wheat seeds. Russ. J. Plant Physiol. 52:793-800.

Sripinyowanich, S., P. Klomsakul, B. Boonburapong, T. Bangyeekhun, T. Asami, H. Gu, T. Buaboocha, and S. Chadchawan. 2010. Exogenous ABA induces salt tolerance in indica rice (Oryza sativa L.): The 
role of $O s P 5 C S 1$ and $O s P 5 C R$ gene expression during salt stress. Environ. Expt. Bot. doi: 10.1016/j.envexpbot.2010.01.009.

Szabados, L. and A. Savouré. 2009. Proline: A multifunctional amino acid. Cell 15:89-97.

Uchida, A., T.J. Andre, and H. Takashi. 2002. Effects of hydrogen peroxide and nitric oxide on both salt and heat stress tolerance in rice. Plant Sci. 163:515-523.

Vendruscolo, E.C.G., I. Schuster, M. Pileggi, C.A. Scapim, H.B.C. Molinari, C.J. Marur, and L.G.E. Vieira. 2007. Stress-induced synthesis of proline confers tolerance to water deficit in transgenic wheat. J. Plant Physiol. 164:1367-1376.

Vogel, R.H. and M.J. Kopac. 1960. Some Properties of Ornithine$\delta$-transaminase from Neurosproa. Biochim. Biophys. Acta 37:539-540.

Xu, Y.F., X.L. Sun, J.W. Jin, and H. Zhou. 2010. Protective effect of nitric oxide on light-induced oxidative damage in leaves of tall fescue. J. Plant Physiol. 167:512-518.

Yazici, I., I. Türken, A.H. Sekmen, and T. Demiral. 2007. Salinity tolerance of purslane (Portulanca oleracea L.) is achieved by enhanced antioxidative system, low level of lipid peroxidation and proline accumulation. Environ. Expt. Bot. 61:49-57.

Yoshiba, Y., T. Kiyosue, T. Katagiri, H. Ueda, T. Mizoguchi, K. Yamaguchi-Shinozaki, K. Wada, Y. Harada, and K. Shinozaki. 1995. Correlation between the induction of a gene for $\Delta^{1}$-pyrroline-5carboxylate synthetase and the accumulation of proline in Arabidopsis thaliana under osmotic stress. Plant J. 7:751-760.

Yoshiba, Y., T. Kiyosue, K. Nalcashima, K. Yamaguchi-Shinozaki, and K. Shinozaki. 1997. Regulation of levels of proline as an osmolyte in plants water stress. Plant Cell Physiol. 38:1095-1102.

Zhang, C.S., Q. Lu, and D.P.S. Verma. 1995. Removal of feedback inhibition of $\Delta^{1}$-Pyrroline-5-carboxylate synthetase, the bifunctional enzyme catalyzing the first two steps of proline biosynthesis in plants. J. Biol. Chem. 270:20491-20496.

Zhang, Y.Y., L.L. Wang, Y.L. Liu, Q. Zhang, Q.P. Wei, and W.H. Zhang. 2006. Nitric oxide enhances salt tolerance in maize seedlings through increasing activities of proton-pump and $\mathrm{Na}^{+} / \mathrm{H}^{+}$antiport in the tonoplast. Planta 224:545-555. 